\title{
Continuous Flow Synthesis of Iron Oxide Nanoparticles Using Water-in-Oil Microemulsion
}

\author{
J. Sopoušek ${ }^{a, b}, *$, J. Pinkas ${ }^{a, b}$, J. Buršík ${ }^{c}$, M. Svoboda ${ }^{c}$, and P. Krásenskýa \\ ${ }^{a}$ Masaryk University, Faculty of Science, Department of Chemistry, Brno, 61137 Czech Republic \\ ${ }^{b}$ Masaryk University, Central European Institute of Technology, CEITEC, Brno, 62500 Czech Republic \\ ${ }^{c}$ Institute of Physics of Materials, Academy of Sciences of the Czech Republic, Brno, 61662 Czech Republic \\ *e-mail: sopousek@mail.muni.cz.
}

Received May 6, 2020; revised July 2, 2020; accepted July 6, 2020

\begin{abstract}
A continuous laminar flow reactor for the synthesis of nanopowder in microemulsion is described. The reactor is suitable for separated handling with nucleation, growth, and stabilization processes. The synthesis of iron oxide nanoparticles was selected as a model case. A water-sodium dodecyl sulphate-cyclohexene system was used as the microemulsion system for dissolving reactive aqueous solution, precursor, and a particle stabilizer. The product was purified and transferred to the aqueous phase. The result was a colloid solution of iron oxide nanoparticles in water of 50-200 $\mathrm{nm}$ in size with a zeta potential ranging from -25 to $-57 \mathrm{mV}$. The product was characterized by UV-VIS spectroscopy, powder XRD, dynamic light scattering, electron microscopy, and electron diffraction. The results showed that water-in-oil microemulsion method is useful for the synthesis of nanopowders to obtain large amounts of stable product.
\end{abstract}

DOI: $10.1134 / \mathrm{S} 1061933 \mathrm{X} 20060174$

\section{INTRODUCTION}

The predominant method of preparation of nanoparticles (NPs) in basic research laboratories is a procedure where individual stages of synthesis are carried out without mass exchange with the environment (closed system, one pot synthesis) [1,2]. The term "batch synthesis of NPs" is used for this procedure. Another opportunity is a synthesis in an open system. A typical example is a synthesis in a flow reactor [35]. The continuous synthesis is mainly used in industry.

The main advantage of the batch synthesis is its simplicity and safety, which is important for the syntheses that deal with hazardous chemicals. The disadvantage is the difficulty of reproduction if nucleation, the shape of the NPs, and the rate of their growth depend on the intensity of mixing and the geometric arrangement.

The advantage of continuous synthesis is that the yield is not limited, nucleation and growth stages can be under precise control, and a narrow distribution of NP sizes can be obtained. However, this is a method requiring higher acquisition costs for equipment and synthesis control. The problem of continuous synthesis is to ensure a rapid mixing of the reaction solutions. Difficulties occur when the flow is laminar [6]. This aspect can be improved by turbulent flow, ultrasound irradiation [7], special mixing chambers or other techniques.
However, such improvements of continuous synthesis may not be sufficient. The use of microemulsion techniques is therefore a promising alternative [8]. The use of so-called water-in-oil microemulsions enables to apply reverse micelles [9]. These objects can be observed in the oil-rich systems if a nonpolar liquid (oil) is mixed with water in the presence of a surfactant. The tiny droplets of water embedded within a surfactant envelope act as microreactors for the synthesis of the particles. The size of the reverse micelles is thermodynamically determined and can be controlled by synthesis parameters including water-to-surfactant molar ratio and temperature [10]. The reverse micelles are commonly formed as nanosized objects [11].

The water-in-oil microemulsions, where oil can be substituted also by non-polar liquid phase, were used for batch synthesis of metal and metal oxide NPs. The reverse micelles were used, for example, to prepare stable iron oxide NP dispersions [1] using sodium dodecyl sulphate (SDS) as a surfactant and cyclohexene as oil phase in alkaline environment of sodium hydroxide. The iron oxide NPs were obtained in microemulsion also by use of various reagents, surfactants, and non-polar solvents [12], [13]. The microemulsion technique was used for the synthesis of the ferrite NPs with spinel structure for ferrofluid applications [14]. The batch microemulsion synthesis was applied also in the synthesis of Pd [15], MnZn [16], NiZn [17], Pd/Pt/Fe [18], and NiZnFe NPs [19]. 


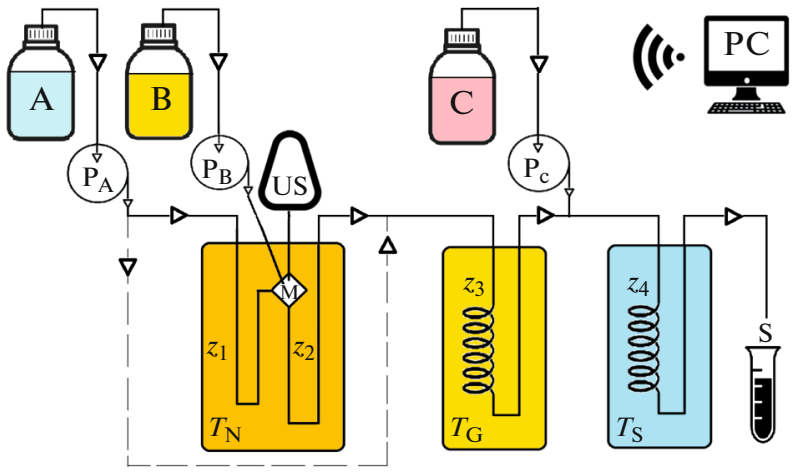

Fig. 1. Schematically shown setup of the continuous laminar flow synthesis of $\mathrm{Fe}_{x} \mathrm{O}_{y}$ NPs using water-in-oil microemulsion. Micellar solutions: A-reaction medium, BFe precursor, $\mathrm{C}-$ stabiliser. $\mathrm{P}_{i}$ - pumps, US-ultrasound source, $\mathrm{Z}_{1}$-preheating to nucleation temperature $T_{\mathrm{N}}$, $\mathrm{M}-$ ultrasound mixing chamber, $\mathrm{Z}_{2}$-nucleation zone, $\mathrm{Z}_{3}$ - growth zone at temperature $T_{\mathrm{G}}, \mathrm{Z}_{4}$-cooling zone to $T_{\mathrm{S}}, \mathrm{S}-$ sampling. $\mathrm{PC}-$ computer to control the synthesis.

The continuous synthesis of NPs was used for example in the one-step preparation of functionalized magnetite nano-flowers in aqueous solution under supercritical conditions [20]. The using of reverse micelles in continuous synthesis is less common; some results for this method are summarized in a review [8]. The magnetic iron NP synthesis differs in a stabilizing substance or in an oil phase [21-23].

The continuous synthesis in reverse micelle systems enables to prepare NPs in a laminar flow. It allows elongation of the reaction times in the order of minutes. The morphology of products differs from those prepared upon applied turbulent flow, where reaction times do not exceed several seconds. This prolongation of the reaction time is promising for better control of the NP nucleation, growth, and functionalization. This aspect is important for the synthesis of functionalized NPs used in diagnostics, sensing, catalysis, and other applications [2].

Herein we describe a laminar flow reactor which was tested on continuous preparation of iron oxide NPs in reverse micelles.

\section{EXPERIMENTAL}

\subsection{Reactor Setup}

The continuous flow reactor (see experimental setup in Fig. 1 and photo in Fig. 2) was proposed for the synthesis of NPs using microemulsion technique. The setup enables to control particle synthesis in a laminar flow. The flow reactor was tested on continuous preparation of iron oxide $\left(\mathrm{Fe}_{x} \mathrm{O}_{y}\right)$ NPs.

The experimental apparatus consists of three main parts: a dosing device, a flow capillary reactor, and a system for the product sampling. The function and setup of synthesis parameters are controlled by software installed on a computer.

The dosing system makes it possible to pump micellar solutions from reservoirs using syringes and peristaltic pumps. Peristaltic pumps are used for dosing the micellar solutions with high consumption

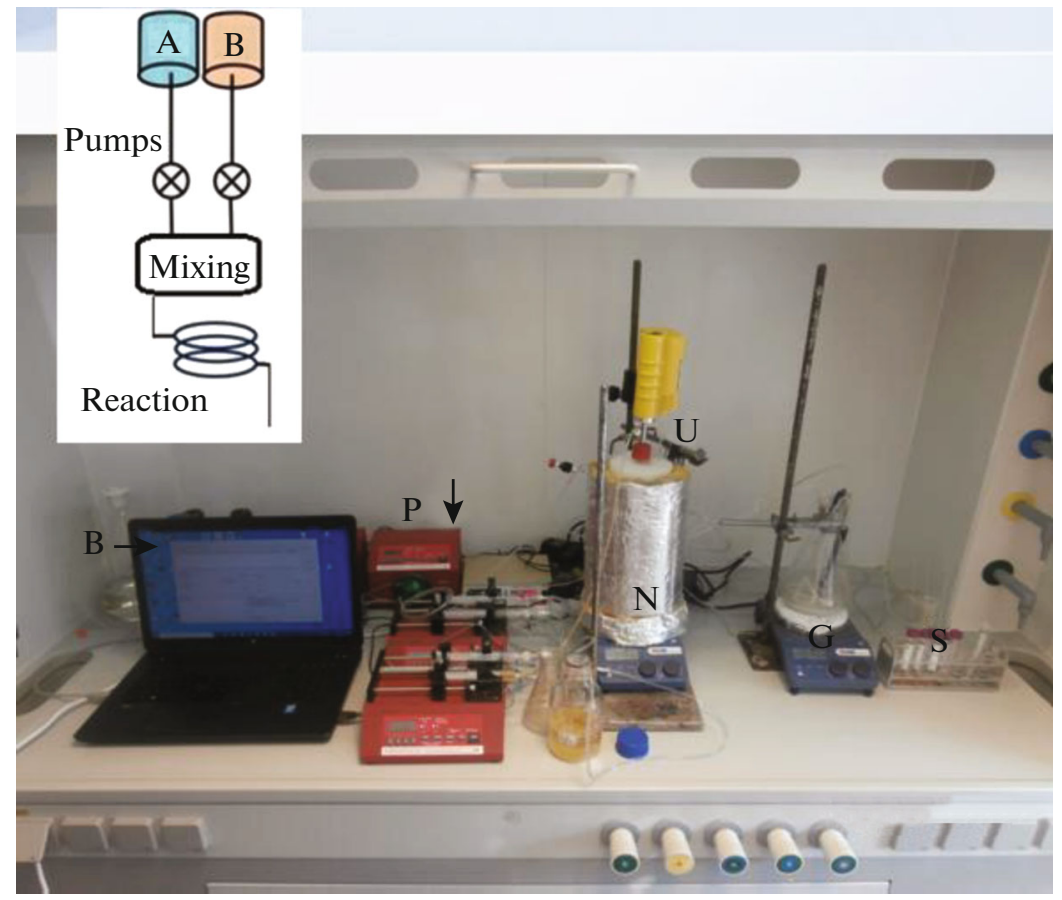

Fig. 2. Digital image of the experimental apparatus described in Fig. 1. 
$\left(0.5-10 \mathrm{~cm}^{3} \mathrm{~min}^{-1}\right)$. Their rate is suitable for dosing a solution containing micelles with a reaction medium.

The syringe pumps are used for dosing micellar solutions of reactants and stabilizer whose consumption volumes are small $\left(0.005-1.0 \mathrm{~cm}^{3} \mathrm{~min}^{-1}\right)$. The system can be extended with extra pumps, if required, for a selected synthesis. The described flow reactor was tested on the preparation of $\mathrm{Fe}_{x} \mathrm{O}_{y}$ NPs. The reverse micellar solution (A) with ammonia was used as the reaction medium, the micellar solution of the $\mathrm{Fe}$ precursor (B) as a reactant, and the micellar citric acid solution $(\mathrm{C})$ as a stabilizer.

The flow capillary reactor has four zones. The first and second zones are located in a thermostat with an adjustable temperature selected for nucleation. The third zone is inside a thermostat set to a temperature, which is optimized for the NP growth. The fourth zone is at a room temperature.

The micellar solution with the reaction medium (A) enters the first zone and is heated to . The preheated solution flows into the second section, which consists of a mixing chamber and a nucleation capillary. The micellar precursor solution (B) is injected into the mixing chamber under ultrasound irradiation. The reverse micelles of both the reaction medium and the precursor are mixed thoroughly. The mixing chamber is a perfectly mixed stream reactor [24] in terms of modelling the reaction kinetics. The contact time $\tau_{\text {mix }}$ can be changed by changing the reaction chamber. During the $\mathrm{Fe}_{x} \mathrm{O}_{y}$ synthesis the contact time was in the range of $0.5-4 \mathrm{~s}$. A laminar flow is established in the nucleation capillary and nuclei of NPs are formed. The nucleation capillary is a tubular reactor. By changing the flow rate, the space time $\tau_{\mathrm{n}}$ can be changed in the range of $20-50 \mathrm{~s}$.

The reaction mixture is cooled down by addition of cold micellar solution (A) and enters the third zone located in a thermostat at temperature. Here, the nuclei grow to form NPs. Spatial time $\tau_{\mathrm{n}}$ reaches $300-$ $1000 \mathrm{~s}$. The micellar stabilizer solution (C) is continuously added when the reaction micellar solution leaves the third zone and the reaction mixture is cooled to the ambient temperature $T_{\mathrm{S}}$.

The system for the product sampling needs to be designed in accordance with the chosen product. The $\mathrm{Fe}_{x} \mathrm{O}_{y}$ NPs can be separated very easily by magnetic field (see Fig. 3).

The setup for the continuous flow synthesis using water-in-oil microemulsions in Fig. 1 enables to optimise the parameters of the synthesis. The temperature in both nucleation and growth zones can be controlled by software. The mixing, nucleation, reaction, and cooling times are given by the flow rate of the micellar solutions by operated pumps. The concentrations of micellar solutions, the volume of the mixing chamber, and the lengths of the capillaries have to be changed manually.

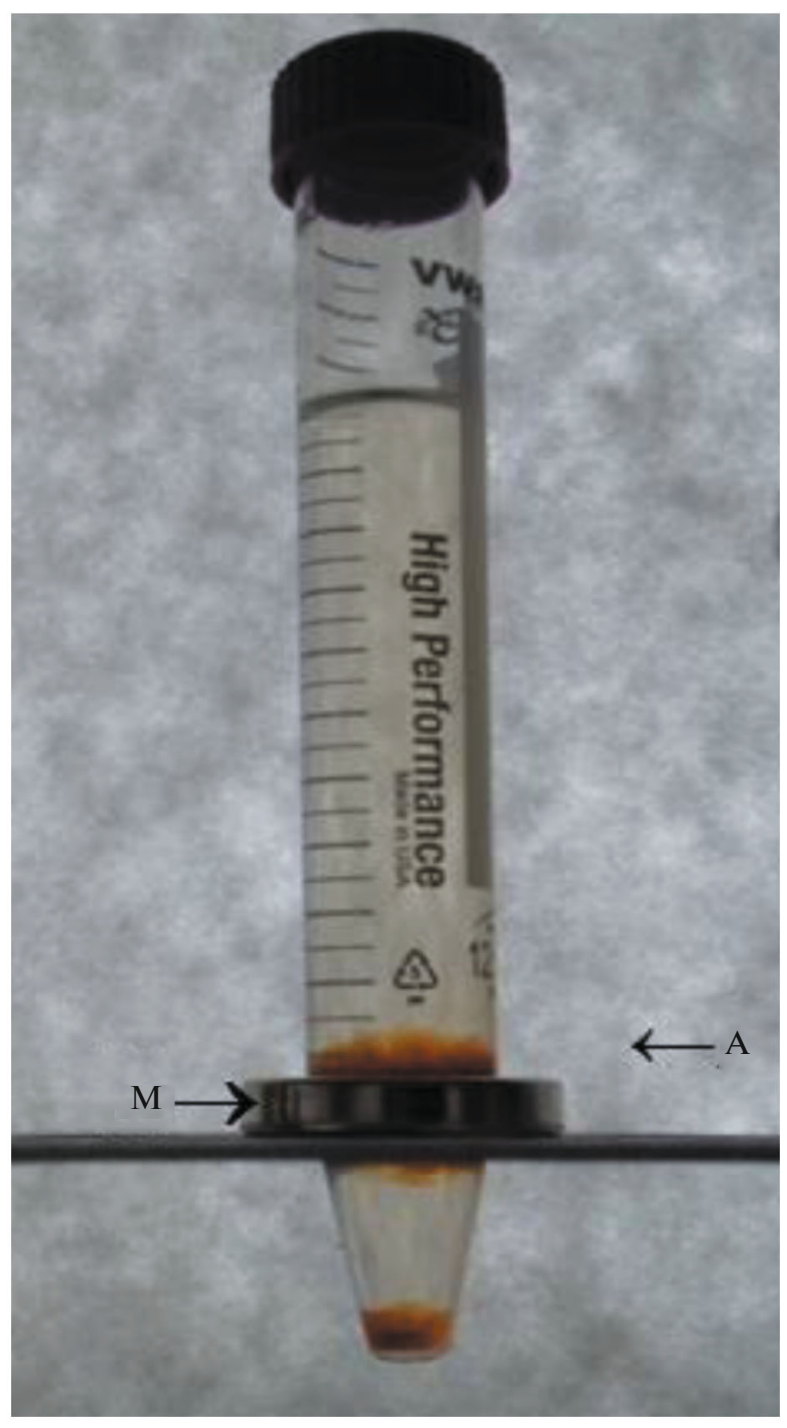

Fig. 3. Optical image of the as-prepared microemulsion with $\mathrm{Fe}_{x} \mathrm{O}_{y}$ NPs. Ring $\mathrm{NdFeB}$ magnet (holding force $11 \mathrm{~kg}$ ) and brown agglomerated NPs attracted by magnetic field are denoted by M and A, respectively.

\subsection{Model Synthesis of Iron Oxide NPS}

The most exploited oxides for the preparation of functionalized iron magnetic NPs are magnetite $\left(\mathrm{Fe}_{3} \mathrm{O}_{4}\right)$ and maghemite $\left(\gamma-\mathrm{Fe}_{2} \mathrm{O}_{3}\right)$. Both oxides have basically a spinel structure and differ mainly in $\mathrm{Fe}(\mathrm{II})$ and $\mathrm{Fe}(\mathrm{III})$ contents [14]. Both magnetite and maghemite have very similar crystal lattices, the distinction between these two compounds by PXRD is difficult because of the broad peaks caused by nanocrystalline substructure [25].

The preparation of $\mathrm{Fe}_{x} \mathrm{O}_{y}$ NPs was chosen as a model synthesis for testing the flow apparatus in Figs. 1 and 2. A significant advantage of this model system is that cheap and safe chemicals are used. The temperatures and times inside zones were controlled. 
The iron oxide synthesis is simple and well examined. To reduce a very long reference list, only the representative reference [26] is given here. The main reaction steps are deprotonation of water molecules coordinated to $\mathrm{Fe}(\mathrm{II})$ or $\mathrm{Fe}$ (III) and dehydration in the alkaline $(\mathrm{pH} \approx 9)$ water solution at $60^{\circ} \mathrm{C}[26]$ under inert gas (mostly nitrogen).

Three initial aqueous solutions were used to obtain the magnetite particles in the experiment described. The first, the water with dissolved ammonia, has been prepared at a level equivalent to ammonia solubility at $60^{\circ} \mathrm{C}$ (about $20 \mathrm{wt} \% \mathrm{NH}_{3}$ ). The second, the mixture of $\mathrm{Fe}(\mathrm{II})$ and $\mathrm{Fe}$ (III) (chlorides or sulphates) in water, was used to create the solution of Fe precursor. The molar ratio of must be kept constant to obtain magnetite particles. The total concentration of Fe used in experiments varied in a range of $0.2-0.8 \mathrm{~mol} \mathrm{dm}^{-3}$. The last water solution contained citric acid (CA) as a stabilising agent with preferred concentration of $0.05 \mathrm{~mol} \mathrm{dm}^{-3}$.

The water/cyclohexene/sodium $n$-dodecyl sulphate microemulsion system was used to prepare $\mathrm{Fe}_{x} \mathrm{O}_{y}$ NPs [13]. The water serves as a solvent of reacting substances: ammonia, Fe precursor, or CA stabiliser. The cyclohexene was used as an oil phase. The sodium $n$-dodecyl sulphate (SDS) served as an anionic surfactant and it was mixed with 1-butanol as co-surfactant in a $1: 1.4$ mass ratio.

The reverse micellar solutions were prepared in subsequent steps. First, the surfactant substances and cyclohexene were mixed in a $1: 1$ mass fraction to create an initial suspension. Next, the portions of the initial suspension were mixed with aqueous solutions of ammonia, Fe precursor or stabiliser in the following weight ratios: 2.4 : 1 (ammonia), $5: 1$ (Fe-precursor), and $4: 1(\mathrm{CA})$. Finally, all reverse micellar solutions were filtered through a rapid flow Malgene SFCA $0.2 \mu \mathrm{m}$ filter.

The prepared reverse micellar solutions of ammonia (solution A), Fe precursor (solution B), and CA stabiliser (solution C) were used in NP synthesis using continuous reactor in Fig. 1. The control system of the flow reactor enabled to vary parameters of the synthesis, mainly the temperatures inside nucleation and growth zones and the flow of solutions $(\mathrm{A}, \mathrm{B}, \mathrm{C})$. The reaction was studied at various concentrations of the substances A, B, and C. The length of the Teflon capillaries inside the nucleation and growth zones was also an alternative for control of the reaction times.

The brown microemulsion of particles interacting with a magnetic field (see Fig. 3) was obtained by the flow synthesis. The iron containing particles were separated from microemulsion by repeated rinsing that consists of centrifugation, removal of the supernatant, and dispersing of the sediment in ethanol. The last operation was to dissolve the sediment in water to obtain a brown colloid solution of the $\mathrm{Fe}_{x} \mathrm{O}_{y}$ particles. These samples were investigated by different methods and used for subsequent studies. The samples were dried to powder, if needed, for subsequent analyses.

The synthesis was conducted to prepare magnetite NPs, but the conditions for purification and phase transport to water were such that oxidation of magnetite to maghemite could be expected [25]. The maghemite NPs are claimed to be less toxic than magnetite NPs for stem cells [27].

The following chemicals were used in the flow synthesis: ammonia aqueous solution $\left(\mathrm{NH}_{3}\right.$, CAS 133621-6, chemical grade: purists p.a., LACHEMA), iron(III) sulphate anhydrous $\left(\mathrm{Fe}_{2}\left(\mathrm{SO}_{4}\right)_{3}\right.$, CAS 1346343-9, purists p.a., LACHEMA), iron(II) sulphate hydrate $\left(\mathrm{Fe}_{2}\left(\mathrm{SO}_{4}\right)_{3} \cdot 7 \mathrm{H}_{2} \mathrm{O}\right.$, CAS 7782-630, purists p. a., LACHEMA), iron(III) chloride anhydrous ( $\mathrm{FeCl}_{3}, \mathrm{CAS}$ 7705-08-0, pure 99\%, LACHNER), iron(II) chloride tetrahydrate $\left(\mathrm{FeCl}_{2} \cdot 4 \mathrm{H}_{2} \mathrm{O}\right.$, CAS 13478-10-9, pure 99\%, LACHNER), citric acid hydrate $\left(\mathrm{C}_{6} \mathrm{H}_{3} \mathrm{O}_{7} \cdot \mathrm{H}_{2} \mathrm{O}\right.$, CAS 59-49-29-1, purists p.a., ONEX), sodium $n$-dodecyl sulphate (SDS, $\mathrm{C}_{12} \mathrm{H}_{25} \mathrm{NaO}_{4} \mathrm{~S}$, CAS 151-21-3, purists p.a., LACHNER), 1-butanol $\left(\mathrm{C}_{4} \mathrm{H}_{10} \mathrm{O}\right.$, CAS 71-36-3, 99.69\%, LACHNER), cyclohexene $\left(\mathrm{C}_{6} \mathrm{H}_{12}\right.$, CAS 110-82-7, purists p.a., LACHNER), water (deionized, $\kappa<$ $1 \mu \mathrm{S} / \mathrm{cm})$, ethanol $\left(\mathrm{C}_{6} \mathrm{H}_{5} \mathrm{OH}\right.$, denatured with about $1 \%$ methyl ethyl ketone).

\subsection{Instrumentation}

Components of continuous flow reactor. New Era Pumps Systems NE-9000 (peristaltic) and NE-4000 (syringe) were used for solution delivery. A Hielscher US Technology homogenizer UP50H was used for ultrasonic mixing. Chemical glass and Teflon capillaries were used to construct the flow reactor. After each experiment, the reactor was cleaned with hydrochloric acid.

Dynamic light scattering (DLS) and $\zeta$ potential measurements. The $\mathrm{Fe}_{x} \mathrm{O}_{y}$ colloid aqueous solutions were investigated by a Zetasizer Nano ZS ZEN 3500 instrument (Malvern, UK) working at the scattering angle of $173^{\circ}$. The method enabled to obtain hydrodynamic size of $\mathrm{Fe}_{x} \mathrm{O}_{y}$ NPs, which is given by both the metal core and the stabilizing organic layer. The same instrument equipped with a DIP cell (Malvern) was used for measurements of the $\zeta$ potential of the $\mathrm{Fe}_{x} \mathrm{O}_{y}$ particles.

Transmission electron microscopy (TEM and HRTEM). The metal cores of the $\mathrm{Fe}_{x} \mathrm{O}_{y}$ particles were investigated by electron microscopy. To prepare samples, a $\mathrm{Fe}_{x} \mathrm{O}_{y}$ powder was placed on a $\mathrm{Cu}$ grid coated by a carbon film. The size and shape of $\mathrm{Fe}_{x} \mathrm{O}_{y}$ particle cores were investigated using a Philips CM12 STEM microscope with a thermoemission source operated at $120 \mathrm{kV}$ and a JEOL JEM 2100F high-resolution TEM (HRTEM) with an FEG source operated at $200 \mathrm{kV}$ (point resolution of $2.3 \AA$ ). Both transmission

$$
\text { COLLOID JOURNAL Vol. } 82 \text { No. } 62020
$$


electron microscopes were equipped with energy dispersive X-ray (EDX) detectors.

Powder X-ray diffraction (PXRD). Measurements were carried out on a GNR Europe XRD 600 diffractometer equipped with a Co lamp, $\lambda\left(K_{\alpha}\right)=1.7903 \AA$, $40 \mathrm{kV}, 15 \mathrm{~mA}$, with $\theta / 2 \theta$ configuration. Samples were measured in step scan of $0.2 \mathrm{deg}$. for $10 \mathrm{~s}$ in a reflection mode on plastic or aluminium sample holders. A 1D detector DECTRIS Mythen2R was used. The samples were treated under air at ambient temperature.

Scanning electron microscopy (SEM). Morphology and overall composition of the samples were observed by scanning electron microscopy (SEM) using a TESCAN LYRA 3XMU FEG/SEM microscope with an X-Max 80 EDX Oxford Instruments detector.

\section{RESULTS AND DISCUSSION}

The apparatus shown in Figs. 1 and 2 was used for the synthesis of $\mathrm{Fe}_{x} \mathrm{O}_{y}$ NPs. Various concentrations of the precursor and stabilizer in the aqueous phases were used for the preparation of micellar solutions.

The concentration of aqueous ammonia solution equivalent to its solubility at the temperature of the nucleation zone $\left(60^{\circ} \mathrm{C}\right)$ was found to be optimal for the process. The advantage of using this method is a simple preparation of the product by heating and degassing the excess of $\mathrm{NH}_{3}$. This heating treatment of the aqueous ammonia solution prevents the formation of a gaseous phase in the apparatus when the reaction medium (A) was preheated to the temperature of nucleation.

The main difficulties in preparing the aqueous phase of the Fe precursor are the oxidation reaction of $\mathrm{Fe}(\mathrm{II})$ to $\mathrm{Fe}$ (III) [28-31] and the hydrolysis of $\mathrm{Fe}$ (III) ions. The oxidation reaction of $\mathrm{Fe}(\mathrm{II})$ changes the $\mathrm{Fe}(\mathrm{III}) / \mathrm{Fe}(\mathrm{II})$ ratio. The molar ratio was controlled before each synthesis via redox potential by using platinum electrode. The optimal potential equivalent to $\mathrm{Fe}(\mathrm{III}) / \mathrm{Fe}(\mathrm{II})=2$ was obtained by extra addition of $\mathrm{Fe}$ (II). Hydrolysis of Fe(III) can only be suppressed if a mixture of $\mathrm{Fe}(\mathrm{III})$ and $\mathrm{Fe}(\mathrm{II})$ with a $\mathrm{pH}<2$ is used for preparation and storage of the micellar solution.

The concentration of the CA solution can be selected over a wide range of about $0.05 \mathrm{~mol} \mathrm{dm}^{-3}$. The stabilizer is very effective due to the strong interaction with the surface of the $\mathrm{Fe}_{x} \mathrm{O}_{y}$ NPs.

Preparation of micellar solutions of reaction medium, $\mathrm{Fe}$ precursor, and stabilizer was performed according to the procedure described in a paragraph 2.2. The obtained solutions were transparent. The formation of reverse micelles was confirmed by a combination of methods. The size of the reverse micelles was measured by DLS. The hydrodynamic radius ranged from 5 to $7 \mathrm{~nm}$, which is comparable to that of $6.9 \mathrm{~nm}$ measured for SDS/heptane/butanol [9]. We did not observe clear dependence of the micelle size on the chemical composition of the aqueous phase.

The feed of the micellar solutions was controlled by settings of the pumps. The inlet of the micellar ammonia solution (A) was chosen so that the contact time in the mixing chamber did not exceed 2 s. This time corresponds to a flow rate of solution (A) > $8 \mathrm{~cm}^{3} \mathrm{~min}^{-1}$. The flow of solution (A) was usually set to $10 \mathrm{~cm}^{3} \mathrm{~min}^{-1}$. The micellar precursor solution (B) inlet was chosen so that the $\mathrm{pH}$ did not drop below 9 in the nucleation zone due to the neutralization reaction between basic solution (A) and acidic solution (B). The optimum inlet rate of micellar solution (B) was about $0.05 \mathrm{~cm}^{3} \mathrm{~min}^{-1}$. When the $\mathrm{pH}$ dropped below 9 , different products were formed (for example, maghemite). The total reaction time for nucleation and growth ranged from 2 to $10 \mathrm{~min}$ depending on the length of the capillaries. The inlet of the CA solution $\mathrm{C}$ was set to $1 \mathrm{~cm}^{3} \mathrm{~min}^{-1}$. The degree of aggregation increases with increasing time between mixing the precursor with the reaction medium and the time of adding the stabilizer. The nucleation and growth temperatures were varied as well. The nucleation temperature had the greatest influence on both size and zeta potential of the produced NPs.

The mixing of the micellar solutions (A) and (B) was performed with the assistance of ultrasound. The power of the $50 \mathrm{~W}$ sonicator and its parameters were the same for all experiments. The ultrasonic source was sufficient for thorough mixing of the micellar solutions of the reaction medium (A) and the Fe precursor (B). It can be assumed that ultrasound affects nucleation. However, the effect was not monitored.

After purification, the aqueous solution of stabilized $\mathrm{Fe}_{x} \mathrm{O}_{y}$ NPs was prepared and characterized by the DLS method. The hydrodynamic size of the prepared NPs was between 50 and $200 \mathrm{~nm}$. The colloid $\mathrm{Fe}_{x} \mathrm{O}_{y}$ NPs samples were evaporated under vacuum at room temperature. The dry powder was analysed by PXRD. The method showed nanocrystallinity of obtained samples. The diffractions of the representative sample are shown in Fig. 4. The size of the crystallites was evaluated according to the Scherrer equation at $5.6 \mathrm{~nm}$ [32].

The application of $\mathrm{CA}$ as the surface stabilizing ligand leads to the changes in the surface charge. The surface charge (zeta potential) was therefore measured. The zeta potential was very sensitive to the experimental setup. The zeta potential of the samples with increased stability was ranging from -25 to $-57 \mathrm{mV}$.

The size, shape, and chemical composition of the $\mathrm{Fe}_{x} \mathrm{O}_{y}$ NPs were investigated in details by TEM and SEM methods. The chemical composition of the powders was determined by the EDX detector. Figure 5 shows a representative example of the $\mathrm{Fe}_{x} \mathrm{O}_{y}$ NPs. A particle with 60-70 nm diameter is composed of smaller crystallites as it was also confirmed by the 


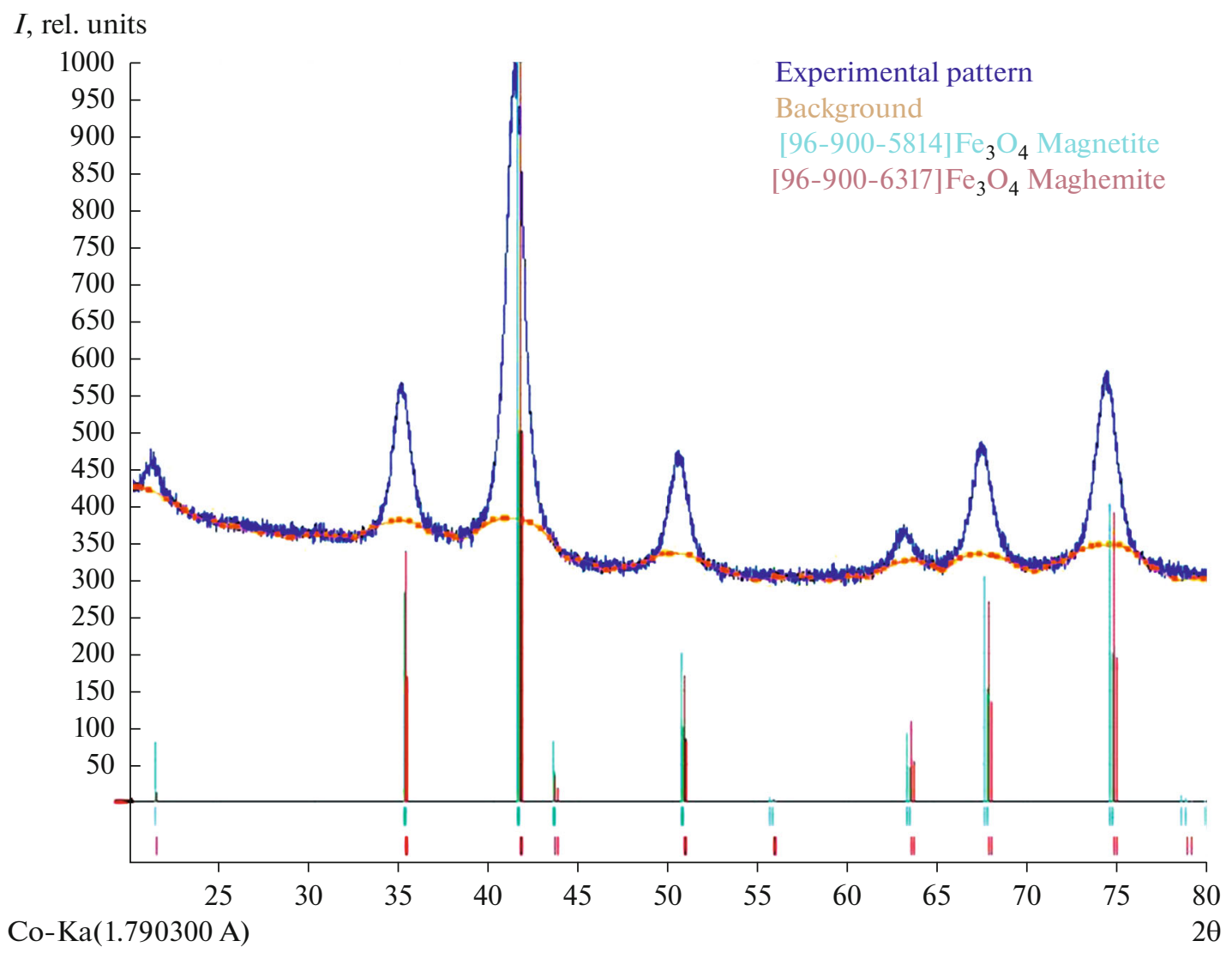

Fig. 4. PXRD data of $\mathrm{Fe}_{x} \mathrm{O}_{y}$ NPs. The crystal size of $5.6 \mathrm{~nm}$ estimated by the Scherrer equation; the crystal lattice corresponds to those of magnetite and maghemite.

PXRD data used for calculation of particle size by the Scherer equation (see Fig. 4). Thus, the dry powders consist of aggregated NPs. The aggregate is constituted by the individual NPs with the size about $5 \mathrm{~nm}$ as it was revealed by the HRTEM (Fig. 6). Similar dopamine stabilized aggregates have been obtained in [22] and [33].

The stability of the $\mathrm{Fe}_{x} \mathrm{O}_{y}$ NPs solution was investigated as a function of nucleation temperature. The study

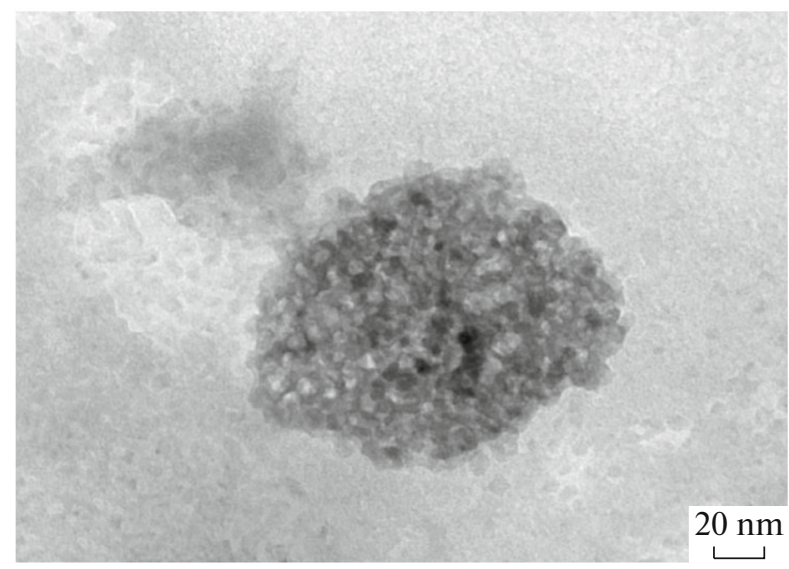

Fig. 5. TEM image of a single $\mathrm{Fe}_{x} \mathrm{O}_{y} \mathrm{NP}$. was performed at different setups. The representative results are given in Figs. 7-9. The conditions used specifically for this study were: a saturated $\mathrm{NH}_{3}$ solution at $60^{\circ} \mathrm{C}$, total $\mathrm{Fe}$ concentration $0.5 \mathrm{~mol} \mathrm{dm}^{-3}$, CA concentration $0.05 \mathrm{~mol} \mathrm{dm}^{-3}, T_{\mathrm{G}}=$ room temperature, and flow rates of $\mathrm{A}-10, \mathrm{~B}-0.05$, and $\mathrm{C}-1 \mathrm{~cm}^{3} \mathrm{~min}^{-1}$.

The product stability of the micellar synthesis using the continuous reactor was monitored at constant

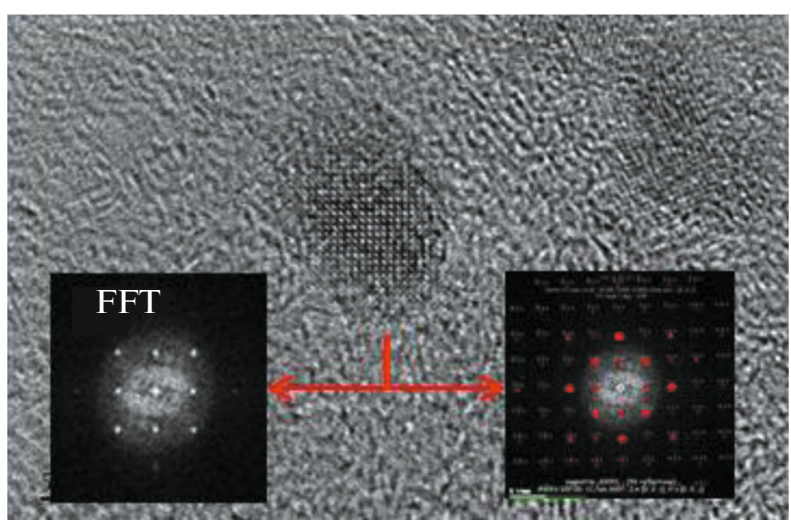

Fig. 6. HRTEM image of a $\mathrm{Fe}_{x} \mathrm{O}_{y}$ nanocrystal with a size $5.2 \mathrm{~nm}$. 


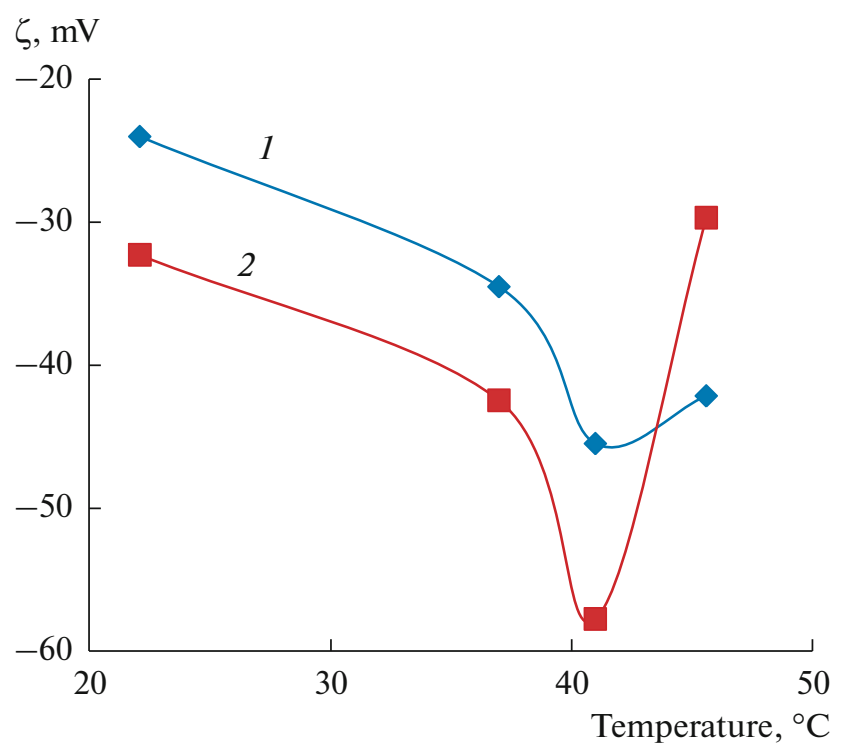

Fig. 7. Values of zeta potential of $\mathrm{Fe}_{x} \mathrm{O}_{y}$ NPs formed at different nucleation temperatures $T_{\mathrm{N}}$ and measured immediately after preparation ( 1 ) and after 3 -day equilibration (2). nucleation temperatures $T_{\mathrm{N}}: 22,37,41$, and $45 \pm$ $0.5^{\circ} \mathrm{C}$. The growing and cooling temperatures $T_{\mathrm{G}}$ and $T_{\mathrm{S}}$ were equal to $22^{\circ} \mathrm{C}$. The most significant effect was the change of zeta potential with the temperature in the nucleation zone $T_{\mathrm{N}}$ (Fig. 7).

The hydrodynamic size and zeta potential of the $\mathrm{Fe}_{x} \mathrm{O}_{y}$ NPs samples in water was studied by the Zetasizer Nano ZS device, which allows to measure the zeta potential with a reproducibility $\pm 0.1 \mathrm{mV}$. The micellar system is sensitive to the preparation procedure of the micellar solutions A, B, and C. When newly-prepared solution was used, the curve in Fig. 7 could shift up within the range of $5 \mathrm{mV}$. However, the minimum at a temperature around $40 \pm 5^{\circ} \mathrm{C}$ remained pronounced. The most stable NPs with the lowest zeta potential were obtained at a temperature $T_{\mathrm{N}}=41^{\circ} \mathrm{C}$. The zeta potential distribution is plotted in Fig. 8.

Samples were re-monitored after 3 days of storing under ambient conditions. As can be seen from the dependencies in Figs. 7 and 8, the stability changed with time. Samples prepared at nucleation temperatures below $40^{\circ} \mathrm{C}$ showed a decrease in zeta potential corresponding to an increase in stability of colloid solution. The sample prepared at $45^{\circ} \mathrm{C}$ showed an increase in zeta potential to a value of $-27 \mathrm{mV}$, which is in a range of -30 to $+30 \mathrm{mV}$ that implies no sufficient electrostatic stabilization.

The size of NPs in the colloidal solution after aging was in agreement with the obtained zeta potential curve. In the case of stable solution with non-aggregated NPs, the size did not change. The colloid solution with low stability formed sediment (see Fig. 9).

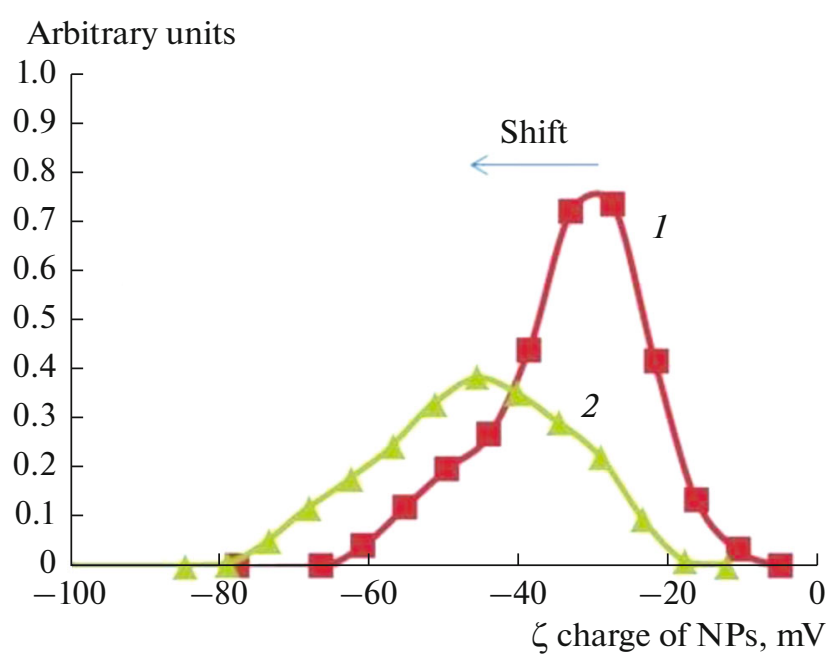

Fig. 8. Zeta potential distribution curves for the aqueous solution of $\mathrm{Fe}_{x} \mathrm{O}_{y}$ NPs prepared at $37^{\circ} \mathrm{C}$ and measured immediately after preparation (1) and after 3-day equilibration (2).

The long-time storage in air led to formation of maghemite sediment in all samples.

\section{CONCLUSIONS}

The laboratory reactor for continuous synthesis of NPs in laminar flow was designed and constructed. Microemulsions containing reverse micelles of reaction medium, precursor and stabilizer were used to

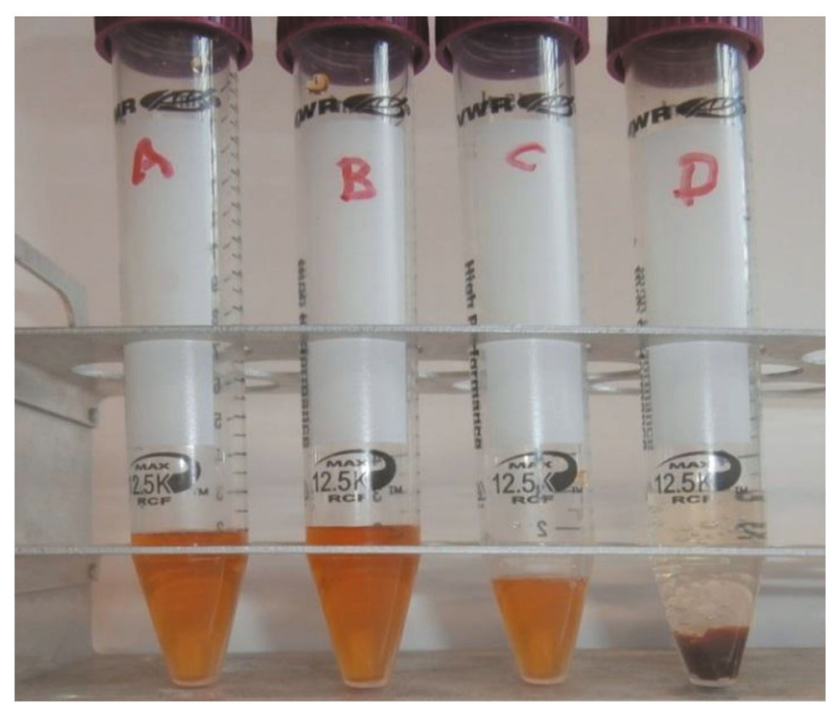

Fig. 9. Optical images of the aqueous solutions of $\mathrm{Fe}_{x} \mathrm{O}_{y}$ NPs after 3-day equilibration. The samples were prepared at different nucleation temperatures $T_{\mathrm{N}}: 22$ (A), 37 (B), $41(\mathrm{C})$, and $45^{\circ} \mathrm{C}(\mathrm{D})$. 
prepare the resulting product. Mixing of the reactants was performed in the chamber with the assistance of ultrasound. The linear reactor consists of three zones with different temperatures, which can be optimized for nucleation growth and stabilization of NPs.

The described continuous laminar flow reactor makes it possible to carry out reactions with reaction times in the order of minutes as opposed to turbulent flow continuous reactors, which are suitable only for rapid reactions. The described reactor is a useful model for the implementation of the NP synthesis into industrial processes.

The laboratory linear reactor was used for the synthesis of $\mathrm{Fe}_{x} \mathrm{O}_{y}$ NPs in laminar flow. The SDS/cyclohexene microemulsion system was used to obtain solutions with reverse micelles. During the experiment the conditions of the synthesis were varied. The influence of the synthetic parameters on the size of $\mathrm{Fe}_{x} \mathrm{O}_{y}$ NPs as well as on their aggregation in aqueous solution was monitored.

The microemulsion continuous reactor presented in this experimental work can help to separate nucleation, growth and stabilization stages of the NP synthesis. This arrangement has not yet been used for the synthesis of metal oxide nanopowder. The method enables to obtain product with better quality then that achieved by use of batch synthesis.

\section{FUNDING}

Financial support of the Grant Academy of Czech Republic of project "Advanced Experimental and Theoretical Approaches to Size-Dependent Phase Diagrams of Nanoalloys" (GA17-15405S) is gratefully acknowledged. This research has also been financially supported by the MEYS CR under the project CEITEC 2020 (LQ1601) and the Horizon 2020 Research and Innovation Program under the grant agreement no. 810626 (SINNCE).

\section{CONFLICT OF INTEREST}

The authors declare that they have no conflict of interest.

\section{REFERENCES}

1. Nourafkan, E., Asachi, M., Gao, H., Raza, G., and Wen, D., J. Ind. Eng. Chem., 2017, vol. 50, p. 57.

2. Jun, Y., Huh, Y.-M., Choi, J., Lee, J.-H., Song, H.-T., Kim, S., Yoon, S., Kim, K.-S., Shin, J.-S., Suh, J.-S., and Cheon, J., J. Am. Chem. Soc., 2005, vol. 127, p. 5732 .

3. Thomas, G., Demoisson, F., Chassagnon, R., Popova, E., and Millot, N., Nanotechnology, 2016, vol. 27, p. 135604.

4. Elbasuney, S. and Mostafa, S.F., Powder Technol., 2015, vol. 278, p. 72.

5. Jung, Y.J., Park, S.H., Song, K.H., and Choe, J., Powder Technol., 2012, vol. 217, p. 325.
6. Bal, V. and Bandyopadhyaya, R., Chem. Eng. J., 2019, vol. 371 , p. 43.

7. Corradi, A.B., Bondioli, F., Ferrari, A.M., Focher, B., and Leonelli, C., Powder Technol., 2006, vol. 167, p. 45.

8. Tovstun, S.A. and Razumov, V.F., Russ. Chem. Rev., 2011, vol. 80, p. 953.

9. Moulik, S.P. and Paul, B.K., Adv. Colloid Interface Sci., 1998, vol. 78, p. 99.

10. Razumov, V.F. and Tovstun, S.A., Colloid J., 2019, vol. 81, p. 337.

11. Ghosh, S., Ghatak, C., Banerjee, C., Mandal, S., Kuchlyan, J., and Sarkar, N., Langmuir, 2013, vol. 29, p. 10066.

12. Darab, J.G., Pfund, D.M., Fulton, J.L., Linehan, J.C., Capel, M., and Ma, Y., Langmuir, 1994, vol. 10, p. 135.

13. Drmota, A., Drofenik, M., Koselj, J., and Znidarsic, A., in Microemulsions - An Introduction to Properties and Applications, Najjar R., Ed., InTech, 2012.

https://doi.org/10.5772/36154.

14. Makovec, D., Košak, A., Žnidaršič, A., and Drofenik, M., J. Magn. Magn. Mater., 2005, vol. 289, p. 32.

15. Perez-Coronado, A.M., Calvo, L., Alonso-Morales, N., Heras, F., Rodriguez, J.J., and Gilarranz, M.A., Colloids Surf. A, 2016, vol. 497, p. 28.

16. Aubery, C., Solans, C., and Sanchez-Dominguez, M., Langmuir, 2011, vol. 27, p. 14005.

17. Uskoković, V. and Drofenik, M., Colloids Surf. A, 2005, vol. 266, p. 168.

18. Abazari, R., Heshmatpour, F., and Balalaie, S., ACS Catal., 2013, vol. 3, p. 139.

19. Dar, M., Shah, J., Siddiqui, W.A., and Kotnala, R.K., Appl. Nanosci., 2014, vol. 4, p. 675.

20. Demoisson, F., Ariane, M., Piolet, R., and Bernard, F., Adv. Eng. Mater., 2011, vol. 13, p. 487.

21. Ahmadi, R., Malek, M., Hosseini, H.R.M., Shokrgozar, M.A., Oghabian, M.A., Masoudi, A., Gu, N., and Zhang, Y., Mater. Chem. Phys., 2011, vol. 131, p. 170.

22. Liao, N., Wu, M., Pan, F., Lin, J., Li, Z., Zhang, D., Wang, Y., Zheng, Y., Peng, J., Liu, X., and Liu, J., Sci. Rep., 2016, vol. 6, p. 18746.

23. Boyer, C., Whittaker, M.R., Bulmus, V., Liu, J., and Davis, T.P., NPG Asia Mater., 2010, vol. 2, p. 23.

24. Hill, C.G. and Root, T.W., Introduction to Chemical Engineering Kinetics and Reactor Design, New York: Wiley, 2014.

25. Barrow, M., Taylor, A., Fuentes-Caparros, A.M., Sharkey, J., Daniels, L.M., Mandal, P., Park, B.K., Murray, P., Rosseinsky, M.J., and Adams, D.J., Biomater. Sci., 2018, vol. 6, p. 101.

26. Gupta, A.K. and Gupta, M., Biomaterials, 2005, vol. 26, p. 3995.

27. Singh, N., Jenkins, G.J.S., Asadi, R., and Doak, S.H., Nano Rev., 2010, vol. 1, p. 5358.

28. Cavasino, F.P., Sbriziolo, C., and Liveri, M.L.T., J. Chem. Soc., Faraday Trans., 1998, vol. 94, p. 395.

29. Gobe, M., Kon-No, K., Kandori, K., and Kitahara, A., J. Colloid Interface Sci., 1983, vol. 93, p. 293.

30. Sinha, M.K., Sahu, S.K., Meshram, P., Prasad, L.B., and Pandey, B.D., Powder Technol., 2015, vol. 276, p. 214.

31. Inouye, K., Endo, R., Otsuka, Y., Miyashiro, K., Kaneko, K., and Ishikawa, T., J. Phys. Chem., 1982, vol. 86 , p. 1465 .

32. Patterson, A.L., Phys. Rev., 1939, vol. 56, p. 978.

33. Wu, M., Zhang, D., Zeng, Y., Wu, L., Liu, X., and Liu, J., Nanotechnology, 2015, vol. 26, p. 115102. 